\title{
Exploring views of orthopaedic surgeons, rheumatologists and general practitioners about osteoarthritis management
}

\author{
Jason A. Wallis ${ }^{1,2,3}$ ( ) Christian J. Barton ${ }^{4,5}$ | Natasha K. Brusco ${ }^{1,6}$ | \\ Joanne L. Kemp ${ }^{4}$ | James Sherwood ${ }^{1}$ | Kirby Young ${ }^{1}$ | Sophie Jennings ${ }^{1}$ | \\ Adrian Trivett $^{7}$ | Ilana N. Ackerman ${ }^{3}$
}

${ }^{1}$ Centre for Allied Health Research and Education, Cabrini Health, Malvern, Victoria, Australia

${ }^{2}$ Monash Department of Clinical Epidemiology, Cabrini Institute, Malvern, Victoria, Australia

${ }^{3}$ Department of Epidemiology and Preventive Medicine, School of Public Health and

Preventive Medicine, Monash University,

Melbourne, Victoria, Australia

${ }^{4}$ La Trobe Sport and Exercise Medicine Research Centre, School of Allied Health, Human Services and Sport, La Trobe

University, Bundoora, Victoria, Australia

${ }^{5}$ Department of Surgery, St Vincent's Hospital, The University of Melbourne, Fitzroy, Victoria, Australia

${ }^{6}$ Rehabilitation, Ageing and Independent Living (RAIL) Research Centre, School of Primary and Allied Health Care, Monash University,

Frankston, Victoria, Australia

${ }^{7}$ Department of Orthopaedic Surgery, Cabrini Health, Malvern, Victoria, Australia

\section{Correspondence}

Jason A. Wallis, Monash Department of Clinical Epidemiology, Cabrini Institute, 4 Drysdale Street, Malvern, VIC 3144, Australia. Email: jwallis@cabrini.com.au and jason. wallis@monash.edu

Funding information

Cabrini Foundation, Grant/Award Number: STO42

\begin{abstract}
Background: Delivery of recommended treatments for hip and knee osteoarthritis $(\mathrm{OA})$ is known to be discordant with guideline recommendations. However, professional views related to OA management across medical and surgical disciplines are not well understood. The aim of this study was to explore the views of medical professionals about management of hip and knee OA.

Methods: Qualitative study. Semistructured individual interviews were conducted with orthopaedic surgeons, rheumatologists and general practitioners routinely involved in the management of OA. Interviews were audiotaped, transcribed, member-checked, coded and thematically analysed.

Results: Fifteen medical professionals were interviewed. Three main themes were: (i) recognition of the importance of nonsurgical management of hip and knee OA, focussed on self-management, exercise-therapy, weight management and analgesia; (ii) joint replacement being considered the 'last resort' for end stage disease not responding to nonsurgical management; and (iii) determination of management 'success' through patient perceptions was more common than the use of validated instruments. Views on management broadly converged across disciplines, except for the role of joint replacement, considered an adjunct in the overall management of OA by rheumatologists and as a definitive cure by orthopaedic surgeons.

Conclusions: Aligning with current guidelines, medical professionals recognised the importance of nonsurgical management focussed on exercise-therapy for hip and knee $\mathrm{OA}$, and concurred that joint replacement surgery should be a last resort. A focus on patient education was less prominent, which along with implementation of validated outcome measures in routine medical practice, may require greater health system support.
\end{abstract}

\section{KEYWORDS}

doctor, hip, knee, osteoarthritis management, qualitative, surgeon

This is an open access article under the terms of the Creative Commons Attribution-NonCommercial-NoDerivs License, which permits use and distribution in any medium, provided the original work is properly cited, the use is non-commercial and no modifications or adaptations are made.

(c) 2021 The Authors. Musculoskeletal Care published by John Wiley \& Sons Ltd. 


\section{1 | INTRODUCTION}

Clinical practice guidelines for hip and knee osteoarthritis (OA) recommend patient education, an active lifestyle and behavioural interventions including exercise-therapy and weight loss management as first line treatments (Bannuru et al., 2019; NICE, 2014; RACGP, 2018). Pharmacological therapies and other passive therapies, such as manual therapy, may be required as second line treatment if there is not an adequate improvement in symptoms and/or function (Roos \& Juhl, 2012). Most of these interventions can be provided by general practitioners, or via referral to allied health professionals including physiotherapists. Rheumatologists may also be required to assist with patient management, particularly in the presence of comorbidities which is a common feature of OA (Muckelt et al., 2020). Hip and knee replacement surgery may be indicated as third-line treatment in the case of severe joint disease, and referral to an orthopaedic surgeon is indicated where there is little improvement after appropriate first and second line management (Victorian Musculoskeletal Clinical Leadership Group, 2018).

In practice, the delivery of recommended treatments for hip and knee $\mathrm{OA}$ is known to be discordant with guideline recommendations (Allen et al., 2017; Nelson et al., 2014). Strongly recommended treatments, such as exercise-therapy and weight loss, are commonly underutilised by people with hip and knee OA (Allen et al., 2017). An Australian study has shown that only $56 \%$ of people with hip or knee OA had previously tried muscle strengthening exercises and $38 \%$ had never tried weight loss (Hinman et al., 2015). The prevalent referral for, and use of, treatments that are not recommended in guidelines is also concerning (Deveza et al., 2018). In the USA between 2007 and 2014 , opioids were prescribed for $13 \%$ of patients with hip OA and $16 \%$ of patients with knee OA (DeMik et al., 2017). Based on prescribing trends, opioid prescriptions for OA in Australia are expected to almost triple over the period 2015/16-2030/31 (Ackerman, Zomer, et al., 2018).

A variety of factors may explain current evidence-to-practice gaps in the management of hip and knee OA at patient, provider and health service levels. Patient-related barriers include treatment costs, misinformed beliefs and motivation (Ackerman et al., 2016; Dobson et al., 2016; Hurley et al., 2018). Health professional-related barriers include knowledge and skills gaps (Briggs et al., 2019; Egerton, Diamond, et al., 2017; Selten et al., 2017). Health servicerelated barriers include inadequate clinical practice guideline dissemination and limited capacity of public and private healthcare services to implement recommendations (Nelson et al., 2014). Currently, similarities and differences in views related to OA management across medical disciplines are not well understood. Previous qualitative evidence of medical professionals' views regarding overall OA management (Alami et al., 2011; Bunzli et al., 2017; Egerton, Nelligan, et al., 2017; Li et al., 2013) has sought views of only one discipline (Egerton, Nelligan et al., 2017; Li et al., 2013) or involved views on specific decision aid tools or interventions to aid management (Alami et al., 2011; Bunzli et al., 2017). Understanding any concordance or discordance between medical professional's views on overall management of OA could inform development of resources to support professional learning and foster optimal communication for the interdisciplinary care for OA.

This qualitative study aimed to explore the views of medical professionals about management of hip and knee OA, across three disciplines commonly involved in contemporary OA management.

\section{2 | METHODS}

\section{1 | Study design}

A qualitative study involving individual semistructured interviews with medical professionals was conducted. A phenomenological approach (Creswell \& Poth, 2016) was applied to explore the participants' subjective perspectives about their views on OA management, based on their clinical experience. The data reported in this paper are drawn from a larger qualitative study exploring patient and medical professional perceptions of the barriers and facilitators around referral to OA management programs (Wallis et al., 2020). The consolidated criterion for reporting qualitative research checklist (COREQ) was used for study reporting (Tong et al., 2007).

\section{2 | Participants}

Medical professionals were eligible to participate in the study if they were: (i) a registered orthopaedic surgeon, rheumatologist or general practitioner and (ii) currently involved in managing patients presenting with hip and/or knee OA. Based on existing clinical networks, a convenience sample of 15 medical professionals meeting these criteria and working at private and/or public hospitals or medical practices in metropolitan Melbourne were recruited and provided consent to participate in the study.

\section{3 | Data collection}

Individual semistructured interviews that explored views related to OA management, and barriers and facilitators to management and referral to nonsurgical management programs was previously published (Wallis et al., 2020). The interview schedule (Table 1) was developed by the authors, based on the theoretical domains framework (Atkins et al., 2017) and refined using the determinants of implementation behaviour questionnaire (Huijg et al., 2014). The current qualitative analysis only examined views related to the management of OA, with open-ended questions and prompts used to ensure participants' own views were elicited, based on their clinical experience. Data collection was undertaken until data saturation was achieved, at which point it was noted that no new themes were emerging (Guest et al., 2006). 
TABLE 1 Interview guide for medical professionals

\begin{tabular}{|c|c|}
\hline Topic areas & Questions (prompts) \\
\hline Management of knee and hip OA & $\begin{array}{l}\text { What role do you consider surgical treatments have in the management of } \\
\text { knee/hip OA? }\end{array}$ \\
\hline & $\begin{array}{l}\text { What role do you consider non-surgical treatments have in the management of } \\
\text { knee/hip OA? (Prompts for specific treatments - exercise-therapy including } \\
\text { resistance, aerobic and functional exercise, education, weight management, } \\
\text { analgesia such as NSAIDS, paracetamol and opioids, injection therapy, gait } \\
\text { aids, taping or braces, electrotherapy agents such as ultrasound, tens, } \\
\text { manual therapy such as mobilisation and massage) }\end{array}$ \\
\hline
\end{tabular}

What components do you think should be included in an appropriate nonsurgical management program for knee/hip OA?

Referrals and recommendations for nonsurgical management for knee/hip OA
What advice do you commonly provide to your patients with knee/hip OA when they first present?

Do you refer your OA patients to other health care professionals or services? If so, to whom?

Under what circumstances would you consider referring for (or recommending a patient receive) non-surgical management of their knee/hip OA? If rheumatologist-under what circumstances would you consider referring for any other nonsurgical or nonmedical management?

What factors do you consider when referring a patient for non-surgical management for their knee/hip OA?

How do you decide where to refer a patient for nonsurgical management of their knee/hip OA?

How would you determine whether a non-surgical management program has been effective for your patient with knee/hip OA?

Knowledge and recommendations for physiotherapy programs

What is your understanding of available physiotherapy programs for knee/hip $\mathrm{OA}$ ?

How many of your patients with knee/hip OA do you recommend attend physiotherapy?

Knowledge of GLA:D® program

What is your understanding of the GLA:D ${ }^{\circledR}$ program?

What is your understanding of the qualifications and experience of the staff who run the $G L A: D \circledast$ program?

Barrier and enablers for referrals

Can you tell me about any factors that would make it easier/or has made it easier to refer into the program?

Can you tell me about any factors that would make it/or has made it difficult to refer into the GLA:D® program? (Prompts included broad options: Referral process, program effectiveness, confidence in intervention and intervention givers, cost versus benefit, access issues for patient, patient preference.)

Describe any difficulties you have encountered (or potentially would encountered) when/if referring your patient to the GLA:D ${ }^{\circledR}$ program?

Are there any aspects of the GLA:D® program you liked? If so please elaborate?

Are there any aspects of the GLA:D® program you disliked? If so please elaborate?

Do you have anything else you would like to add about the management of knee/hip OA?

Abbreviations: GLA:D ${ }^{\circledR}$, Good Life with osteoArthritis in Denmark, NSAIDS, nonsteroidal anti-inflammatory drugs, OA, osteoarthritis.

The interviewer (JS), a registered physiotherapist of 9 years' experience, worked in the same clinical network as most of the medical professionals interviewed in this study. All interviews were conducted face to face, either within quiet rooms on hospital wards or medical consulting suites within the hospital and external to the hospital. All interviews were audiotaped, transcribed verbatim and reviewed line by line by the interviewer. The interviewer received training in qualitative interviewing techniques from an experienced 
qualitative researcher (IA). Member-checking was also undertaken, providing each participant an opportunity to review their transcript for accuracy and this process did not result in any significant changes to the content of the transcripts.

\section{4 | Data analysis}

Qualitative analysis of interview data commenced with a close review of each transcript by three researchers (JW, CB, IA). Second, an inductive thematic analysis (Braun \& Clarke, 2006) was used to code the interview data. This was supported by NVivo software (QSR International Ptd, Ltd.) with one researcher (CB) conducting the initial coding and a second researcher (IA) independently coding a random sample of $50 \%$ of the interview data. Emergent themes were discussed between three researchers until a consensus was reached. The three researchers (JW, CB, IA) were experienced physiotherapists (range 14-22 years' experience) and researchers (range 1-11 years' post $\mathrm{PhD}$ ), and all had previous experience in qualitative data analysis.

\section{3 | RESULTS}

\section{1 | Participant characteristics}

Fifteen medical professionals (six orthopaedic surgeons, four rheumatologists and five general practitioners) were interviewed. The average length of the whole interview was $20 \mathrm{~min}$ (range 14-30 min). Participants had an average age of 52 years (SD 12) and 25 years of clinical experience (SD 15). Table 2 reports the demographic and employment characteristics of the participants.

\section{2 | Themes}

Three main themes were identified: (i) recognition of the importance of non-surgical management of hip and knee OA, focussed on selfmanagement, exercise-therapy, weight management and analgesia; (ii) joint replacement being considered the 'last resort' for end stage disease not responding to non-surgical management; and (iii) determination of management 'success' through patient perceptions rather than the use of validated instruments. The following text summarises the emergent themes with illustrative quotes. Additional illustrative quotes are presented in Table S1.

(i) Recognition of the importance of non-surgical management of hip and knee OA, focussed on self-management, exercise-therapy, weight management and analgesia.

All participants considered that nonsurgical management, particularly self-management, exercise-therapy and analgesia, was important for patients with hip and knee OA and should be provided before considering surgery. The perceived value was summed up by one rheumatologist:

Critical, critical. And by that, I don't mean just medications and injection. I think strength training in particular is something that's very underutilised. Rheumatologist (Participant 2)

Nonsurgical management was broadly interpreted to incorporate tailored treatment options, guided by both clinical symptoms and radiological features. Treatments considered to be important included encouragement to self-manage, improve lifestyle and keep mobile, activity modification (commonly reported by orthopaedic surgeons), physiotherapy, exercise-therapy, weight management, analgesia (including injections), heat/ice/compression (for acute exacerbations), glucosamine despite acknowledging no evidence of efficacy, manual therapy, use of gait aids and braces and management of mental health.

I usually like people to modify their activities, I like them to lose weight, I like them to stop running, jogging, and doing activities that stir things up. Orthopaedic surgeon (Participant 4)

The most valuable components of nonsurgical management were perceived to be exercise-therapy, weight management and analgesia. Exercise-therapy was considered important for varying reasons, including providing more muscular support for joints, improving confidence about activities and mobility and the potential to facilitate positive surgical outcomes. Weight management was considered important by all medical professionals interviewed, yet challenges in achieving weight loss were acknowledged. Medical professionals also talked about the regular provision of patient education and the importance of making patients understand their own responsibility in treatment. Several medical professionals also recognised that education can be challenging to deliver effectively due to time constraints (e.g., general practice consultations) and patient language barriers.

Firstly, I think we have to look at lifestyle issues, whether they're moving, whether got this weight on their joints, weight loss, and their diets, generally, whether they're actually mobile at home, whether they can have some mild analgesia like Panadol to help them along but education, very important. General practitioner (Participant 7)

Simple analgesia was considered important to control pain and keep people mobile. Paracetamol was preferred over antiinflammatories due to being safer, but anti-inflammatories were suggested to produce better results. Medical professionals typically recommended avoiding opioids where possible. Cortisone injections played a common role in management, particularly if surgery was not considered appropriate to achieve short-term improvement in 
TABLE 2 Participant demographics

\begin{tabular}{|c|c|}
\hline Variable & $n=15$ \\
\hline \multicolumn{2}{|l|}{ Profession, $n(\%)$} \\
\hline GP & $5(33)$ \\
\hline Rheumatologist & $4(27)$ \\
\hline Orthopaedic surgeon & $6(40)$ \\
\hline Age (years), mean (SD) & $52(12)$ \\
\hline Female, $n(\%)$ & $1(7)$ \\
\hline Years practicing, mean (SD) & $25(15)$ \\
\hline GP & $32(15)$ \\
\hline Rheumatologist & $22(14)$ \\
\hline Orthopaedic surgeon & $15(10)$ \\
\hline \multicolumn{2}{|c|}{ Current volume of management of hip and knee OA, mean (SD) } \\
\hline High volume ${ }^{a}$ & $13(87)$ \\
\hline Low volume & $2(13)$ \\
\hline \multicolumn{2}{|c|}{ Work setting for orthopaedic surgeons and rheumatologists, $n \%$} \\
\hline Both private and public & $8(80)$ \\
\hline Private only & $2(20)$ \\
\hline Public only & $0(0)$ \\
\hline
\end{tabular}

Abbreviations: GP, general practitioner; OA, osteoarthritis; SD, standard deviation.

'High volume included following examples of responses-'all or majority of practice', 'a lot', 'daily', '75 to $90 \%$ ', 'at least $30-40$ patients per week'.

symptoms (e.g., before travelling), for the treatment of acute exacerbations and based on patient preference. Other injectables such as hyaluronic acid and platelet rich plasma were commonly used or trialled despite participants being aware that these treatments lacked evidence of effectiveness.

Steroid injection of knees, I occasionally do. It's just analgesia of various kinds. Sometimes if they request plateletrich plasma or Synvisc or - what's the other one? Stem cells, etcetera. I generally discourage them and left it up to the patient. Rheumatologist (Participant 15)

(ii) Joint replacement being considered the 'last resort' for end stage disease not responding to non-surgical management.

Joint replacement was perceived by participants to be important for people with severe, end stage OA. It was a common view that joint replacement should be offered to address severe pain and/or disability for people who were not responding to appropriate nonsurgical management.

When considering the appropriateness of joint replacement surgery, surgeons considered a range of factors including age and fitness for surgery, patient willingness to undergo surgery, expectations about the outcome from surgery and likelihood of postoperative complications. For example, one orthopaedic surgeon's view was that younger patients with higher function were less likely to be satisfied with a joint replacement and consequently emphasised the importance of ensuring appropriate non-surgical options were appropriately trialled before surgery was offered.

$$
\begin{aligned}
& \text { So the younger you are when you have a knee replacement } \\
& \text { and the more function you've got when you have a knee } \\
& \text { replacement, the less likely you are to love the knee } \\
& \text { replacement 'cause it's an artificial knee. Orthopaedic } \\
& \text { surgeon (Participant 6) }
\end{aligned}
$$

Divergent views between rheumatologists and orthopaedic surgeons regarding the role of joint replacement surgery were evident. Two rheumatologists referred to joint replacement surgery as an 'adjunct' treatment, with a role in the overall management of OA, while two orthopaedic surgeons considered that joint replacement was the 'ultimate surgery' and a 'definitive cure' in cases of severe disease. Surgeons also discussed the appropriateness of other surgical procedures for knee OA including osteotomies, patellofemoral joint replacements and arthroscopy. It was the view of some orthopaedic surgeons that osteotomies may have a role in knee OA management for younger patients to help prevent OA progression, that patellofemoral surgery was a difficult procedure and may not help and knee arthroscopy was not a useful procedure for OA. 
(iii) Determination of management 'success' through patient perceptions rather than use of validated instruments.

Most participants relied on the patient's overall perception of improvement in symptoms, function, physical activity and satisfaction to determine treatment success, rather than the use of validated, disease-specific outcome instruments. Only one orthopaedic surgeon and one rheumatologist reported using patient-reported outcome measures to assist in decision making about surgery and to judge treatment success.

Happy patients, pretty simple. Orthopaedic surgeon (Participant 4)

Oh, they'll tell me if they're better or not. General practitioner (Participant 7)

Common considerations for treatment success included pain levels, activity, subjectively reported quality of life, individual goals or overall satisfaction with care. Activity examples included walking distance, ease of using stairs, work, travel, the ability to provide care to others and to look after themselves.

\section{DISCUSSION}

This study provides unique insight into similarities and differences in views related to hip and knee OA management across relevant medical disciplines, including orthopaedic surgeons, rheumatologists and general practitioners. As a positive finding, all 15 experienced medical professionals interviewed in this study valued the importance of nonsurgical management, especially self-management, exercisetherapy, analgesia and weight management, and considered surgical management as a last resort. However, a key indicator of treatment success from most participants was patient perception of improvement and satisfaction, and not validated patient-reported outcome measures. Diverging views regarding the role of joint replacement, albeit acknowledged by all participants as a final treatment step, likely reflects the different perspectives of the medical specialities. These findings support health professional learning and encourage communication between all members of the treating team for the optimal interdisciplinary care for people with OA.

The perceived importance of nonsurgical treatments for hip and knee OA, including exercise-therapy and weight management, aligns with recommendations across international clinical practice guidelines (Bannuru et al., 2019; NICE, 2014). However, some medical professionals interviewed in this study considered that exercisetherapy and weight management were interventions not particularly 'done well' from a holistic health service perspective. This is consistent with reported breakdowns in the translation of guideline recommendations to clinical practice (Nelson et al., 2014) and the limited resources for clinicians and patients to access, particularly for noninsured patients within the public health system (Ackerman et al., 2016). Implementing strategies and supports to overcome barriers and to leverage facilitators, in order to facilitate recommended treatments is needed. International initiatives to develop and implement guideline-based nonsurgical OA management programs (ESCAPE-pain, 2020; GLA:D®, 2020; Jönsson et al., 2019) provide an accessible treatment option and are beginning to help bridge these evidence-to-practice gaps, but more health system reform may also be needed to improve funding and enhance accessibility for patients (Caneiro et al., 2020).

Provision of patient education, which is known to be an important first line OA treatment for enhancing understanding about OA and its optimal management (Bannuru et al., 2019; NICE, 2014; RACGP, 2018), was raised by most participants and implies this is considered an important component of routine care. However, patient education was rarely emphasised as a focus of treatment, and delivering effective patient education was considered challenging by participants due to time constraints within medical consultations. Educational interventions align closely with the skill set and scope of practice of allied health professionals (such as physiotherapists), who could play a greater role in shared education delivery, in conjunction with their medical colleagues. Additionally, educational interventions that focus specifically on behavioural change (e.g. motivational interviewing and cognitive behavioural therapy), can be used to support optimal management by encouraging participation in exercise-therapy and engagement with weight management strategies for this population (Hinman et al., 2017).

General practitioners, rheumatologists and surgeons in this study typically recommended medical and surgical management that is consistent with contemporary clinical guidelines such as the judicious use of nonsteroidal anti-inflammatories, with avoidance of opioids and knee arthroscopy (ACSQHC, 2019; Bannuru et al., 2019; RACGP, 2018). One pharmacological treatment that was commonly viewed favourably by medical professionals in this study was cortisone injections. This was recommended with certainty and perceived short term benefit, without consideration that it might be harmful to cartilage health (McAlindon et al., 2017). Some study participants also used non-recommended interventions while recognising a lack of evidence to guide clinical practice with certainty (e.g., platelet rich plasma injections) or known limited efficacy (e.g., paracetamol, glucosamine). Surgical procedures such as osteotomies were also considered to have a role in management of younger patients by some of the orthopaedic surgeons despite a lack of evidence suggesting a need for research related to these treatments (Brouwer et al., 2014). Uncertain evidence commonly exists for OA treatments and presents challenges for health professionals, requiring good communication skills to assist their patients to make the best treatment decisions consistent with their patients' contextual and environmental factors (Bunzli et al., 2019).

There was limited use of validated patient-reported outcome measures (PROMs) to evaluate the success of treatment, despite a mounting international push to adopt the use of these tools in clinical practice (Ackerman et al., 2018). PROMs can be used to monitor patient progress following treatment, facilitate patient-clinician 
communication, inform shared decision making and patient-centred care and support interdisciplinary communication between health professionals (Ackerman et al., 2018; Snyder et al., 2012). However, most of the medical professionals interviewed in this study based their determination of treatment success on their patient's overall perception of improvement and satisfaction, rather than PROMs, possibly reflecting beliefs that integrating PROMs in clinical practice can be challenging (Ackerman et al., 2018). While our findings indicate a need to support medical professionals to implement routine PROMs into clinical practice, some health services prioritise collection of PROMs to evaluate surgical interventions for hip and knee osteoarthritis (Tew et al., 2020). Also, a pilot implementation of PROMs data collection was recently undertaken by the Australian Orthopaedic Association National Joint Replacement Registry (AOANJRR, 2020). As this pilot is implemented nationally, orthopaedic surgeons may become more comfortable with embedding collection of PROMs within their practice.

Consistent with clinical guideline recommendations (NICE, 2014; RACGP, 2018), all medical professionals in this study considered joint replacement surgery to be the last resort and only following an appropriate trial of nonsurgical management. An interesting finding was that joint replacement was considered as an 'adjunct' in the overall management of OA by rheumatologists, compared to a 'definitive cure' by orthopaedic surgeons. This divergent view may be explained by different training and clinical experiences for the two medical specialities. Replacement of osteoarthritic joint surfaces may be perceived as the 'definitive cure' for management of end-stage OA by surgeons due to superior benefits for pain, function and quality of life compared to nonsurgical care alone (Learmonth et al., 2007; Skou et al., 2018). However, from a rheumatologist perspective, joint replacement may be considered an 'adjunct' for the holistic management of the patient with OA, with their broader focus on the whole person's health (Australian Rheumatology Association, 2020). While joint replacement demonstrates benefits to joint pain and quality of life (Learmonth et al., 2007; Skou et al., 2015), it may not address conditions associated with their OA such as systemic inflammation (Goldring \& Otero, 2011), poor cardiovascular health (Nüesch et al., 2011), low levels of physical activity (Harding et al., 2014) and complications from surgery may arise.

A strength of this study was its focus on key stakeholder views from three clinical disciplines that routinely provide OA care in both primary care and speciality settings. Robust qualitative research methods were used (including member checking of transcripts and independent data review) and the interview schedule was developed by an experienced research team with longstanding clinical experience in OA management. A limitation of this study is, by its nature, that qualitative research has limited generalisability beyond the study sample, but rather it is used to generate detailed personal perspectives that cannot be obtained through quantitative methods. We also acknowledge the possibility of responder bias, whereby participants with greater knowledge of contemporary evidence and clinical guidelines may have been more likely to participate in the study.

\section{CONCLUSION}

Aligning with current guidelines, medical professionals recognised the importance of nonsurgical management focussed on exercisetherapy for hip and knee OA and concurred that joint replacement surgery should be a last resort. A focus on patient education was less prominent, which along with implementation of validated PROMs in routine medical practice may require greater health systems support. These findings support health professional learning and encourage communication between all members of the treating team for the optimal interdisciplinary care for people with OA.

\section{ACKNOWLEDGEMENTS}

The authors would like to acknowledge all the participants in this trial and their willingness to participate in this research. This research received competitive grant funding from the Cabrini Foundation. The study sponsors had no role in the study design; in the collection, analysis and interpretation of data; in the writing of the manuscript; and in the decision to submit the manuscript for publication.

\section{CONFLICT OF INTERESTS}

The authors declare that there are no conflict of interests.

\section{AUTHOR CONTRIBUTIONS}

Jason A. Wallis made a substantial contribution to conception and study design, analysis and interpretation of data, drafting the manuscript and revising it critically for important intellectual content. Ilana N. Ackerman, Christian J. Barton and Natasha K. Brusco made substantial contributions to conception and study design, analysis and interpretation of data and revising the manuscript critically for important intellectual content. James Sherwood made a substantial contribution to the acquisition of interview data, analysis and interpretation of data and revising the manuscript critically for important intellectual content. Joanne L. Kemp, Kirby Young, Sophie Jennings and Adrian Trivett made substantial contributions to analysis and interpretation of data, and revising the manuscript critically for important intellectual content. All authors read and approved the version of the manuscript to be published, and agreed to be accountable for all aspects of the work in ensuring that questions related to the accuracy or integrity of any part of the work are appropriately investigated and resolved.

\section{DATA AVAILABILITY STATEMENT}

Interview data from this study are not available for sharing given potential confidentiality implications associated with smaller participant samples and consistent with current ethical approvals.

\section{ETHICS STATEMENT}

Ethics approval was obtained from the Cabrini Health Human Research Ethics Committee (reference no. 02-22-01-18) in compliance with the Helsinki declaration. All participants provided written informed consent. 


\section{ORCID}

Jason A. Wallis (D) https://orcid.org/0000-0003-4740-2149

\section{REFERENCES}

Ackerman, I. N., Cavka, B., Lippa, J., \& Bucknill, A. (2018). The feasibility of implementing the ICHOM standard set for hip and knee osteoarthritis: A mixed-methods evaluation in public and private hospital settings. The Journal of Patient-Reported Outcomes, 2(1), 32.

Ackerman, I. N., Livingston, J. A., \& Osborne, R. H. (2016). Personal perspectives on enablers and barriers to accessing care for hip and knee osteoarthritis. Physical Therapy, 96(1), 26-36.

Ackerman, I. N., Zomer, E., Gilmartin-Thomas, J.-M., \& Liew, D. (2018). Forecasting the future burden of opioids for osteoarthritis. Osteoarthritis and Cartilage, 26(3), 350-355.

ACSQHC. (2019). Osteoarthritis of the knee clinical care standard. Retrieved from https://www.safetyandquality.gov.au/publicationsand-resources/resource-library/osteoarthritis-knee-clinical-care-sta ndard

Alami, S., Boutron, I., Desjeux, D., Hirschhorn, M., Meric, G., Rannou, F., \& Poiraudeau, S. (2011). Patients' and practitioners' views of knee osteoarthritis and its management: A qualitative interview study. PloS One, 6(5), e19634.

Allen, K. D., Golightly, Y. M., \& White, D. K. (2017). Gaps in appropriate use of treatment strategies in osteoarthritis. Best Practice \& Research Clinical Rheumatology, 31(5), 746-759.

AOANJRR. (2020). PROMs pilot report. Retrieved from https://aoanjrr. sahmri.com/proms-pilot-report

Atkins, L., Francis, J., Islam, R., O'Connor, D., Patey, A., Ivers, N. Michie, S. (2017). A guide to using the theoretical domains framework of behaviour change to investigate implementation problems. Implementation Science, 12(77), 1-18.

Australian Rheumatology Association. (2020). What is a Rheumatologist? Retrieved from https://rheumatology.org.au/patients/what-is-arheumatologist.asp

Bannuru, R. R., Osani, M. C., Vaysbrot, E. E., Arden, N. K., Bennell, K., Bierma-Zeinstra, S. M. A. Bhandari, M. (2019). OARSI guidelines for the non-surgical management of knee, hip, and polyarticular osteoarthritis. Osteoarthritis and Cartilage, 27(11), 1578-1589.

Braun, V., \& Clarke, V. (2006). Using thematic analysis in psychology. Qualitative Research in Psychology, 3(2), 77-101.

Briggs, A. M., Houlding, E., Hinman, R. S., Desmond, L. A., Bennell, K. L., Darlow, B. Larmer, P. J. (2019). Health professionals and students encounter multi-level barriers to implementing high-value osteoarthritis care: A multi-national study. Osteoarthritis and Cartilage, 27(5), 788-804.

Brouwer, R. W., Huizinga, M. R., Duivenvoorden, T., van Raaij, T. M., Verhagen, A. P., Bierma-Zeinstra, S. M., \& Verhaar, J. A. (2014). Osteotomy for treating knee osteoarthritis. Cochrane Database of Systematic Reviews, 12, CD004019. https://doi.org/10.1002/146518 58.CD004019.

Bunzli, S., Nelson, E., Scott, A., French, S., Choong, P., \& Dowsey, M. (2017). Barriers and facilitators to orthopaedic surgeons' uptake of decision aids for total knee arthroplasty: A qualitative study. BMJ open, 7(11), e018614.

Bunzli, S., O'Brien, P., Ayton, D., Dowsey, M., Gunn, J., Choong, P., \& Manski-Nankervis, J.-A. (2019). Misconceptions and the acceptance of evidence-based nonsurgical interventions for knee osteoarthritis. A qualitative study. Clinical Orthopaedics and Related Research, 477(9), 1975-1983.

Caneiro, J. P., Roos, E. M., Barton, C. J., O'Sullivan, K., Kent, P., Lin, I., \& O'Sullivan, P. (2020). It is time to move beyond 'body region silos' to manage musculoskeletal pain: Five actions to change clinical practice. British Journal of Sexual Medicine, 54, 8.
Creswell, J. W., \& Poth, C. N. (2016). Qualitative inquiry and research design: Choosing among five approaches (2nd ed.). Sage Publications.

DeMik, D. E., Bedard, N. A., Dowdle, S. B., Burnett, R. A., McHugh, M. A., \& Callaghan, J. J. (2017). Are we still prescribing opioids for osteoarthritis?. The Journal of Arthroplasty, 32(12), 3578-3582.

Deveza, L. A., Hunter, D. J., \& Van Spil, W. E. (2018). Too much opioid, too much harm. Osteoarthritis and Cartilage, 26(3), 293-295.

Dobson, F., Bennell, K. L., French, S. D., Nicolson, P. J., Klaasman, R. N., Holden, M. A. Hinman, R. S. (2016). Barriers and facilitators to exercise participation in people with hip and/or knee osteoarthritis: Synthesis of the literature using behavior change theory. American Journal of Physical Medicine \& Rehabilitation, 95(5), 372-389.

Egerton, T., Diamond, L., Buchbinder, R., Bennell, K., \& Slade, S. C. (2017). A systematic review and evidence synthesis of qualitative studies to identify primary care clinicians' barriers and enablers to the management of osteoarthritis. Osteoarthritis and Cartilage, 25(5), 625638.

Egerton, T., Nelligan, R., Setchell, J., Atkins, L., \& Bennell, K. L. (2017). General practitioners' perspectives on a proposed new model of service delivery for primary care management of knee osteoarthritis: A qualitative study. BMC Family Practice, 18, 85.

ESCAPE-pain. (2020). Enabling self-management and coping with arthritic pain using exercise. Retrieved from https://escape-pain.org/.

$G L A: D \circledast$. (2020). GLA:D® International network. Retrieved from https:// gladinternational.org/home/

Goldring, M. B., \& Otero, M. (2011). Inflammation in osteoarthritis. Current Opinion in Rheumatology, 23(5), 471-478.

Guest, G., Bunce, A., \& Johnson, L. (2006). How many interviews are enough? An experiment with data saturation and variability. Field Methods, 18(1), 59-82.

Harding, P., Holland, A. E., Delany, C., \& Hinman, R. S. (2014). Do activity levels increase after total hip and knee arthroplasty?. Clinical Orthopaedics and Related Research, 472(5), 1502-1511.

Hinman, R. S., Lawford, B. J., Campbell, P. K., Briggs, A. M., Gale, J., Bills, C. Bennell, K. L. (2017). Telephone-delivered exercise advice and behavior change support by physical therapists for people with knee osteoarthritis: Protocol for the telecare randomized controlled trial. Physical Therapy, 97(5), 524-536.

Hinman, R. S., Nicolson, P. J. A., Dobson, F. L., \& Bennell, K. L. (2015). Use of nondrug, nonoperative interventions by community-dwelling people with hip and knee osteoarthritis. Arthritis Care \& Research, 67(2), 305-309.

Huijg, J. M., Gebhardt, W. A., Dusseldorp, E., Verheijden, M. W., van der Zouwe, N., Middelkoop, B. J., \& Crone, M. R. (2014). Measuring determinants of implementation behavior: Psychometric properties of a questionnaire based on the theoretical domains framework. Implementation Science, 9(33), 1-15.

Hurley, M., Dickson, K., Hallett, R., Grant, R., Hauari, H., Walsh, N. Oliver, S. (2018). Exercise interventions and patient beliefs for people with hip, knee or hip and knee osteoarthritis: A mixed methods review. Cochrane Database of Systematic Reviews, 4, CD010842.

Jönsson, T., Eek, F., Dell'Isola, A., Dahlberg, L. E., \& Ekvall Hansson, E. (2019). The better management of patients with osteoarthritis program: Outcomes after evidence-based education and exercise delivered nationwide in Sweden. PloS One, 14(9), e0222657.

Learmonth, I. D., Young, C., \& Rorabeck, C. T. (2007). The operation of the century: Total hip replacement. The Lancet, 370(9597), 1508-1519.

Li, C. S., Pathy, R., Adili, A., Avram, V., Barasi, M. A., Mundi, R. Bhandari, M. (2013). Is the treatment gap in knee osteoarthritis real? A qualitative study of surgeons' perceptions. Journal of Long-Term Effects of Medical Implants, 23, 223-240.

McAlindon, T. E., LaValley, M. P., Harvey, W. F., Price, L. L., Driban, J. B., Zhang, M., \& Ward, R. J. (2017). Effect of intra-articular triamcinolone vs saline on knee cartilage volume and pain in patients with 
knee osteoarthritis: A randomized clinical trial. Jama, 317(19), 19671975.

Muckelt, P. E., Roos, E. M., Stokes, M., McDonough, S., Grønne, D. T., Ewings, S., \& Skou, S. T. (2020). Comorbidities and their link with individual health status: A cross-sectional analysis of 23,892 people with knee and hip osteoarthritis from primary care. Journal of Comorbidity, 10, 1-11.

Nelson, A. E., Allen, K. D., Golightly, Y. M., Goode, A. P., \& Jordan, J. M. (2014). A systematic review of recommendations and guidelines for the management of osteoarthritis: The chronic osteoarthritis management initiative of the US bone and joint initiative. Seminars in Arthritis and Rheumatismm, 43(6), 701-712.

NICE. (2014). Osteoarthritis: Care and management. https://www.nice.org. uk/guidance/cg177

Nüesch, E., Dieppe, P., Reichenbach, S., Williams, S., Iff, S., \& Jüni, P. (2011). All cause and disease specific mortality in patients with knee or hip osteoarthritis: Population based cohort study. BMJ, 342, d1165.

RACGP. (2018). Guideline for the management of knee and hip osteoarthritis (2nd ed.). RACGP. https://www.racgp.org.au/download/Documents/ Guidelines/Musculoskeletal/guideline-for-the-management-of-knee -and-hip-oa-2nd-edition.pdf

Roos, E. M., \& Juhl, C. B. (2012). Osteoarthritis 2012 year in review: Rehabilitation and outcomes. Osteoarthritis and Cartilage, 20(12), 1477-1483.

Selten, E. M., Vriezekolk, J. E., Nijhof, M. W., Schers, H. J., van der MeulenDilling, R. G., van der Laan, W. H. van den Ende, C. H. (2017). Barriers impeding the use of non-pharmacological, non-surgical care in hip and knee osteoarthritis: The views of general practitioners, physical therapists, and medical specialists. Journal of Clinical Rheumatology, 23(8), 405-410.

Skou, S. T., Roos, E. M., Laursen, M. B., Rathleff, M. S., Arendt-Nielsen, L., Rasmussen, S., \& Simonsen, O. (2018). Total knee replacement and non-surgical treatment of knee osteoarthritis: 2-year outcome from two parallel randomized controlled trials. Osteoarthritis and Cartilage, 26(9), 1170-1180.

Skou, S. T., Roos, E. M., Laursen, M. B., Rathleff, M. S., Arendt-Nielsen, L., Simonsen, O., \& Rasmussen, S. (2015). A randomized, controlled trial of total knee replacement. New England Journal of Medicine, 373(17), 1597-1606.

Snyder, C. F., Aaronson, N. K., Choucair, A. K., Elliott, T. E., Greenhalgh, J., Halyard, M. Y., Hess, R., Miller, D.M. Reeve, B.B., \& Santana, M. (2012). Implementing patient-reported outcomes assessment in clinical practice: A review of the options and considerations. Quality of Life Research, 21, 1305-1314.

Tew, M., Dalziel, K., Clarke, P., Smith, A., Choong, P. F., \& Dowsey, M. (2020). Patient-reported outcome measures (PROMs): Can they be used to guide patient-centered care and optimize outcomes in total knee replacement?. Quality of Life Research, 29, 3273-3283.

Tong, A., Sainsbury, P., \& Craig, J. (2007). Consolidated criteria for reporting qualitative research (COREQ): A 32-item checklist for interviews and focus groups. International Journal for Quality in Health Care, 19(6), 349-357.

Victorian Musculoskeletal Clinical Leadership Group. (2018). Victorian model of care for osteoarthritis of the hip and knee. https://www.msk. org.au/wp-content/uploads/2018/07/MoC_Final-report.pdf

Wallis, J. A., Ackerman, I. N., Brusco, N. K., Kemp J., Sherwood, J., Young K., Jennings S., Trivett A., Barton C. J. Barriers and enablers for referral to and participation in a contemporary osteoarthritis management program. Osteoarthritis Cartilage Open, 2020, 2(4), 1-7.

\section{SUPPORTING INFORMATION}

Additional supporting information may be found online in the Supporting Information section at the end of this article.

How to cite this article: Wallis JA, Barton CJ, Brusco NK, et al. Exploring views of orthopaedic surgeons, rheumatologists and general practitioners about osteoarthritis management. Musculoskeletal Care. 2021;1-9. https://doi.org/10.1002/msc.1549 


\section{University Library}

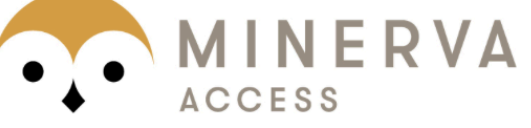

A gateway to Melbourne's research publications

Minerva Access is the Institutional Repository of The University of Melbourne

\section{Author/s:}

Wallis, JA;Barton, CJ;Brusco, NK;Kemp, JL;Sherwood, J;Young, K;Jennings, S;Trivett, A;Ackerman, IN

Title:

Exploring views of orthopaedic surgeons, rheumatologists and general practitioners about osteoarthritis management

Date:

2021-03-12

Citation:

Wallis, J. A., Barton, C. J., Brusco, N. K., Kemp, J. L., Sherwood, J., Young, K., Jennings, S., Trivett, A. \& Ackerman, I. N. (2021). Exploring views of orthopaedic surgeons, rheumatologists and general practitioners about osteoarthritis management. MUSCULOSKELETAL CARE, 19 (4), pp.524-532. https://doi.org/10.1002/msc. 1549.

Persistent Link:

http://hdl.handle.net/11343/274094

License:

CC BY-NC-ND 